\title{
A SPECTRAL ANALYSIS APPROACH FOR ACOUSTIC RADIATION FROM COMPOSITE PANELS
}

\author{
Travis L. Turner* \\ NASA Langley Research Center \\ Hampton, VA 23681-0001
}

\author{
Mahendra P. Singh ${ }^{\dagger}$ \\ Virginia Polytechnic Institute \\ and State University \\ Blacksburg, VA 24061-0219
}

\author{
Chuh $\mathrm{Mei}^{\ddagger}$ \\ Old Dominion University \\ Norfolk, VA 23529-0247
}

\begin{abstract}
A method is developed to predict the vibration response of a composite panel and the resulting farfield acoustic radiation due to acoustic excitation. The acoustic excitation is assumed to consist of obliquely incident plane waves. The panel is modeled by a finite element analysis and the radiated field is predicted using Rayleigh's integral. The approach can easily include other effects such as shape memory alloy (SMA) fiber reinforcement, large deflection thermal postbuckling, and non-symmetric SMA distribution or lamination. Transmission loss predictions for the case of an aluminum panel excited by a harmonic acoustic pressure are shown to compare very well with a classical analysis. Results for a composite panel with and without shape memory alloy reinforcement are also presented. The preliminary results demonstrate that the transmission loss can be significantly increased with shape memory alloy reinforcement. The mechanisms for further transmission loss improvement are identified and discussed.
\end{abstract}

\footnotetext{
Aerospace Engineer, Structural Acoustics Branch, Member AIAA.

$\dagger \quad$ Professor, Engineering Science and Mechanics Department.

$\ddagger \quad$ Professor, Aerospace Engineering Department, Associate Fellow AIAA.

Copyright (c) 1995 by the American Institute of Aeronautics and Astronautics, Inc. No copyright is asserted in the United States under Title 17, U.S. code. The U.S. Government has a royalty-free license to exercise all rights under the copyright claimed herein for Government purposes. All other rights are reserved by the copyright owner.
}

\section{$\underline{\text { Introduction }}$}

Interior or cabin noise is one important issue in the development and design of advanced subsonic and supersonic aircraft. Conventional aircraft typically employ passive treatments, such as constrained layer damping and acoustic absorption materials, to reduce the structural response and resulting acoustic levels in the aircraft interior. These techniques require significant addition of mass and only attenuate relatively high frequency noise transmitted through the fuselage. Improved reduction in the transmitted noise at the lower frequencies is required for advanced subsonic aircraft. Reductions will also be imperative, along with the need for improved fatigue resistance without significant mass addition, for proposed supersonic transports, where the excitation levels will be high due to engine noise and turbulent boundary layer fluctuating pressures.

Although structural acoustic coupling is in general very important in the study of noise transmission through an aircraft fuselage, analysis of noise transmission through a panel supported in an infinite rigid baffle (separating two semi-infinite acoustic domains) can be useful for studies involving active/adaptive materials, complex loading, etc. Transmission of noise through conventional baffled panels has been studied extensively ${ }^{1}$. More recent work has been aimed at developing active and/or adaptive methods of controlling the structural acoustic response of panels to reduce the transmitted noise ${ }^{2}$. These methods have focused on using piezoelectric materials, and to a lesser extent shape memory alloys, to control the structural acoustic response of panels subject to harmonic acoustic excitation.

Turner et al. ${ }^{3}$ developed a finite element formulation to study the response of shape memory alloy hybrid composite panels due to thermal and acoustic loads. A finite element approach was used to allow for 
the SMA material nonlinearity, thermal expansion effects, geometric nonlinearities due to thermal buckling, and complicated geometries and boundary conditions.

The purpose of the present work is to develop an approach for analyzing the structural acoustic response of panels due to obliquely-incident plane-wave acoustic excitation of general temporal nature, with the intent for subsequent studies involving SMA hybrid composite panels. The method is validated through comparison with a classical approach for harmonic excitation ${ }^{1}$. Results are also presented for composite panels with and without shape memory alloy reinforcement.

\section{Shape Memory Alloy Hybrid Composites}

Shape memory alloys exhibit a characteristic phase transformation from martensite to austenite, initiating at the austenite start temperature $T_{s}$ and asymptotically ending at the austenite finish temperature $T_{f}$. A shape memory alloy in the low temperature martensitic condition $\left(T<T_{s}\right)$, when plastically deformed and the external stresses removed, will regain its original (memory) shape when heated. For example, strains of typically six to eight percent can be completely recovered by heating the nickel-titanium alloys (Nitinol) above the austenite finish temperature. The transformation temperatures can be altered by changing the composition of the alloy. In addition, when Nitinol is heated, the Young's modulus increases three to four times and the yielding strength also increases approximately ten times ${ }^{4-7}$.

The materials, referred to in this study as SMA fiber-reinforced hybrid composites, are conventional advanced composite materials (such as graphite-epoxy) that contain embedded SMA fibers having the same direction as the graphite fibers. The memory effect of the SMA fibers may be put to use by applying an initial elongation to them during fabrication of the laminate. Thus, once the laminate is heated above $T_{s}$, the SMA fibers will try to recover the initial strain and induce tensile inplane forces in the laminate. The overall stiffness of the hybrid composite panel will be increased due to: 1) the increase of the Young's modulus of the SMA fibers by a factor of three or four, and 2) the internal tensile inplane forces induced in the panel from the recovery of initial strains of the SMA fibers. Therefore, the root-mean-square (RMS) maximum deflection due to acoustic pressure loading, will be reduced at elevated temperatures as compared to composite panels without embedded SMA fibers.

Shape memory alloys have been applied as actuators for active control of buckling of beams ${ }^{8}$ and shape control of beams ${ }^{9}$. It is also being studied for use in active vibration control of beams ${ }^{10,11}$ and large space structures ${ }^{12}$. Active vibration control of flexible linkage mechanisms using SMA fiber-reinforced composites has been investigated by Venkatesh et al. ${ }^{13}$. Acoustic transmission and radiation control through the use of SMA fibers in a hybrid composite was presented by Liang et al. ${ }^{14}$ and Anders et al. ${ }^{15}$.

\section{Finite Element Formulation}

The finite element equations governing the response of a composite panel, with shape memory alloy wire reinforcement and subject to thermal and acoustic loads, have the form ${ }^{3}$

$$
\begin{gathered}
{\left[\begin{array}{cc}
M_{b} & 0 \\
0 & M_{m}
\end{array}\right]\left\{\begin{array}{c}
\ddot{W}_{b} \\
\ddot{W}_{m}
\end{array}\right\}+\left(\left[\begin{array}{cc}
K_{b} & K_{B} \\
K_{B}^{T} & K_{m}
\end{array}\right]+\left[\begin{array}{cc}
K_{\sigma}^{*} & 0 \\
0 & 0
\end{array}\right]\right.} \\
-\left[\begin{array}{cc}
K_{N \Delta T} & 0 \\
0 & 0
\end{array}\right]+\frac{1}{2}\left[\begin{array}{cc}
N 1_{N m}+N 1_{N B} & N 1_{b m} \\
N 1_{m b} & 0
\end{array}\right] \\
\left.+\frac{1}{3}\left[\begin{array}{cc}
N 2_{b} & 0 \\
0 & 0
\end{array}\right]\right)\left\{\begin{array}{c}
W_{b} \\
W_{m}
\end{array}\right\}=\left\{\begin{array}{c}
P_{b}(t) \\
0
\end{array}\right\}+\left\{\begin{array}{c}
P_{b \Delta T} \\
P_{m \Delta T}
\end{array}\right\} \\
-\left\{\begin{array}{c}
P_{b \sigma}^{*} \\
P_{m \sigma}^{*}
\end{array}\right\}
\end{gathered}
$$

or

$$
\begin{gathered}
{[M]\{\ddot{W}\}+\left([K]+\left[K_{\sigma}^{*}\right]-\left[K_{N \Delta T}\right]+\frac{1}{2}[N 1]\right.} \\
\left.+\frac{1}{3}[N 2]\right)\{W\}=\{P(t)\}+\left\{P_{\Delta T}\right\}-\left\{P_{\sigma}^{*}\right\}
\end{gathered}
$$

where $[M]$ and $[K]$ are the system mass and stiffness matrices; $\left[K_{\sigma}^{*}\right]$ and $\left[K_{N \Delta T}\right]$ are the geometric stiffness matrices due to the SMA recovery stress $\sigma_{r}^{*}$ (or $\left\{N_{\sigma}^{*}\right\}$ ) and thermal inplane force vector $\left\{N_{\Delta T}\right\}$, respectively; [N1] and [N2] are the first and second order nonlinear stiffness matrices which depend linearly and quadratically upon displacement $\{W\}$, respectively; $\{P(t)\}$ is the acoustic excitation load vector, $\left\{P_{\Delta T}\right\}$ is the thermal load vector, and $\left\{P_{\sigma}^{*}\right\}$ is the SMA recovery force vector. The subscripts $b$ and $m$ denote bending and membrane components, respectively; and the subscripts $B, N m$ and $N B$ indicate that the corresponding stiffness matrix is due to the laminate stiffness $[B]$, membrane forces $\left\{N_{m}\right\}\left(=[A]\left\{\epsilon_{m}^{o}\right\}\right)$ and $\left\{N_{B}\right\}(=[B]\{\kappa\})$, respectively. Detailed derivations of the equations of motion and expressions for the element matrices and load vectors are referred to Locke $^{16,17}$.

Consider a rectangular composite panel supported in an infinite rigid baffle, subjected to an incident acoustic pressure on one side and allowed to radiate to 
a semi-infinite acoustic field on the other. The acoustic excitation load vector in equation 2 has the form

$$
\{P(t)\}=\left\{\begin{array}{c}
P_{b}(t) \\
P_{m}(t)
\end{array}\right\}
$$

with

$$
\left\{P_{b}(t)\right\}_{e}=\int_{A_{e}} p(\xi, \eta, t)\left\{F_{w}(\xi, \eta)\right\} d A_{e}
$$

and

$$
\left\{P_{m}(t)\right\}=\{0\}
$$

where the $F_{w}$ are the out-of-plane interpolation functions, $(\xi, \eta)$ are the coordinates in the plane of the plate, and the subscript $e$ indicates an element quantity. The element contributions from equation 4 are assembled to form $\left\{P_{b}(t)\right\}$ for the system. In general, the pressure loading on the panel consists of the incident pressure, the reflected pressure, and the transmitted pressure:

$$
p(\xi, \eta, t)=p_{i}(\xi, \eta, t)+p_{r}(\xi, \eta, t)-p_{t}(\xi, \eta, t)
$$

However, $p(\xi, \eta, t)$ can be written in terms of the blocked pressure and the radiated pressure, as suggested by Roussos ${ }^{1}$. The blocked pressure is that pressure on the incident side when the panel is considered rigid and the radiated pressure is that due to panel motion. The acoustic radiation problem can be solved separately from the forced response of the panel by assuming that the radiated pressure is negligible compared to the blocked pressure. Thus, the load vector $\left\{P_{b}(t)\right\}$ is due to the blocked pressure $\left(p(\xi, \eta, t)=p_{b}(\xi, \eta, t)=2 p_{i}(\xi, \eta, t)\right)$ and the transmitted pressure (pressure radiated to the free field) can be determined from the resulting panel motion, without the need for a fully coupled analysis.

Three different types of analyses are required to determine the response of SMA hybrid composite panels to an acoustic pressure at elevated temperatures. They are: (1) thermal buckling analysis, (2) thermal postbuckling analysis, and (3) vibration analysis of flat or thermally buckled panels. The governing equations for the linear dynamic response of such a panel, assuming that the stable static configuration has been determined, are given by ${ }^{3}$

$$
[M]\{\ddot{W}\}+[\bar{h}]\{W\}=\{P(t)\}
$$

where $[\bar{K}]$ is the stiffness matrix for the flat or buckled panel. The natural frequencies $\omega_{r}$ and mode shapes $\left\{\phi_{r}\right\}$ of vibration are obtained from the eigenvalue problem

$$
\omega_{r}^{2}[M]\left\{\phi_{r}\right\}=[\bar{K}]\left\{\phi_{r}\right\}
$$

A set of uncoupled modal equations with reduced degrees of freedom can thus be obtained from equation 7 as

$$
\lceil I\rfloor\{\ddot{q}\}+\left\lceil 2 \zeta_{r} \omega_{r}\right\rfloor\{\dot{q}\}+\left\lceil\omega_{r}^{2}\right\rfloor\{q\}=\{f(t)\}
$$

where $r=1,2, \ldots, m$ is the number of modes retained, the delimiters \lceil\rfloor indicate a diagonal matrix, and $\lceil I\rfloor$ is the identity matrix, indicating a mass normalized modal matrix. In equations 9, a modal damping term has been included where $\zeta_{r}$ is the modal damping ratio. The physical degrees of freedom can be recovered from the truncated modal transformation

$$
\{W\}=\sum_{r=1}^{m}\left\{\phi_{r}\right\} q_{r}=[\phi]\{q\} \quad m \leq N_{1}
$$

where $N_{1}$ is the total number of degrees of freedom.

The modal response, solution to the modal equations of motion (equations 9), can be determined by time domain analysis or frequency domain analysis. In either case, a continuous or discrete approach can be employed. In this study, analysis is done in the frequency domain through the use of a discrete technique. This allows complicated frequency distributions to be handled in a straightforward manner.

\section{Discrete Spectral Analysis}

A discrete spectral analysis approach was chosen since continuous approaches are limited to rather simple loading cases, which are not very realistic for practical applications and not easily generated in an experimental situation. In practice, there is always a finite amount of data. Thus, one would employ a Finite Fourier Transform to a sampled time history as seen through a viewing window. Furthermore, for discretely sampled time data, a Discrete Fourier Transform (DFT) can be used to estimate the Finite Fourier Transforms, etc. A particularly efficient means of performing a DFT is known as the Fast Fourier Transform (FFT). Of greater importance is that most experimental data is processed via the FFT. Thus, a means of using experimentally measured data has obvious beneficial implications.

\section{Panel Response}

A discrete spectral analysis can be employed by assuming that the modal force vector, and thus the modal response, can be written as the sum of harmonic functions:

$$
\{f(t)\}=\sum_{n=0}^{N_{2}-1}\left\{\hat{f}_{n}\right\} e^{i \omega_{n} t}
$$


and

$$
\{q(t)\}=\sum_{n=0}^{N_{2}-1}\left\{\hat{q}_{n}\right\} e^{i \omega_{n} t}
$$

where $N_{2}$ is the number of discrete frequencies in the transformation and $\left\{\hat{f}_{n}\right\}$ and $\left\{\hat{q}_{n}\right\}$ are the complex spectrum amplitudes vectors, one for each frequency ${ }^{18}$. Substitution into the modal equations of motion, equation 9 , gives

$$
\begin{aligned}
\sum_{n=0}^{N_{2}-1}\left(-\omega_{n}^{2}\lceil I\rfloor\right. & +i \omega_{n}\left\lceil 2 \zeta_{r} \omega_{r}\right\rfloor \\
& \left.+\left\lceil\omega_{r}^{2}\right\rfloor\right)\left\{\hat{q}_{n}\right\} e^{i \omega_{n} t}=\sum_{n=0}^{N_{2}-1}\left\{\hat{f}_{n}\right\} e^{i \omega_{n} t}
\end{aligned}
$$

Therefore, for any one of the discrete frequencies $\omega_{n}$ the relation becomes

$$
\left(\left\lceil-\omega_{n}^{2}\right\rfloor+\left\lceil i 2 \zeta_{r} \omega_{r} \omega_{n}\right\rfloor+\left\lceil\omega_{r}^{2}\right\rfloor\right)\left\{\hat{q}_{n}\right\}=\left\{\hat{f}_{n}\right\}
$$

The modal forcing vector is related to the acoustic excitation load vector from the system diagonalization, equation 9 :

$$
\{f(t)\}=[\phi]^{T}\{P(t)\} \quad \text { or } \quad\left\{\hat{f}_{n}\right\}=[\phi]^{T}\left\{\hat{P}_{n}\right\}
$$

Furthermore, the nonzero elements of that vector result from $\left\{P_{b}(t)\right\}$. The incident pressure is assumed to consist of obliquely incident plane waves, so the incident pressure spectral amplitude vector for an element at a given frequency can be derived from equation 4 and becomes

$$
\begin{aligned}
\left\{\hat{P}_{n}\right\}_{e} & =\int_{A_{e}} 2 \hat{p}_{n}\left\{F_{w}(\xi, \eta)\right\} e^{-i \omega_{n} \frac{(\xi \kappa+\eta \lambda)}{c}} d A_{e} \\
& =\{\Gamma\}_{e} \hat{p}_{n}
\end{aligned}
$$

where $\{\Gamma\}_{e}$ contains the integrated element spatial description of the incident acoustic pressure, $\hat{p}_{n}$ is a spectral amplitude of the acoustic pressure, $\kappa=$ $\sin \theta_{i} \cos \phi_{i}$, and $\lambda=\sin \theta_{i} \sin \phi_{i}$, see figure 1. Therefore, assembly of the element contributions from equation 16 results in

$$
\left\{\hat{f}_{n}\right\}=[\phi]^{T}\{\Gamma\} \hat{p}_{n}
$$

The relation in equation 14 is simply a set of uncoupled algebraic equations for the modal displacement spectral amplitudes. Thus, the modal displacement spectral amplitude vector becomes

$$
\begin{aligned}
\left\{\hat{q}_{n}\right\} & =\left\lceil H\left(\omega_{n}\right)\right\rfloor\left\{\hat{f}_{n}\right\} \\
& =\left\lceil H\left(\omega_{n}\right)\right\rfloor[\phi]^{T}\{\Gamma\} \hat{p}_{n}
\end{aligned}
$$

where the $\left\lceil H\left(\omega_{n}\right)\right\rfloor$ is a diagonal matrix of frequency response functions given by

$$
H_{r}\left(\omega_{n}\right)=\frac{1}{\omega_{r}^{2}-\omega_{n}^{2}+i 2 \zeta_{r} \omega_{r} \omega_{n}}
$$

Note that from equation 12 , the displacement, velocity, and acceleration time responses can be computed by an inverse FFT, assuming that the phase information has been preserved, from

$$
\begin{aligned}
& \{q(t)\}=\sum_{n=0}^{N_{2}-1}\left\{\hat{q}_{n}\right\} e^{i \omega_{n} t} \\
& \{\dot{q}(t)\}=\sum_{n=0}^{N_{2}-1} i \omega_{n}\left\{\hat{q}_{n}\right\} e^{i \omega_{n} t} \\
& \{\ddot{q}(t)\}=\sum_{n=0}^{N_{2}-1}-\omega_{n}^{2}\left\{\hat{q}_{n}\right\} e^{i \omega_{n} t}
\end{aligned}
$$

If the excitation is deterministic, the panel dynamic response solution is complete. However, for stochastic excitation, a statistical approach must be used. Additionally, panel response cross-correlation quantities are of interest for the acoustic radiation discussion to follow in the next section. Therefore, the necessary relations for stochastic panel response are developed subsequently.

The modal displacement response correlation matrix can be formed from equation 12 as

$$
\begin{aligned}
{\left[R_{q q}\left(t_{1}, t_{2}\right)\right] } & =E\left[\left\{q\left(t_{1}\right)\right\}\left\{q\left(t_{2}\right)\right\}^{T}\right] \\
& =\sum_{m=0}^{N_{2}-1} \sum_{n=0}^{N_{2}-1} E\left[\left\{\hat{q}_{m}^{*}\right\}\left\{\hat{q}_{n}\right\}^{T}\right] e^{i\left(\omega_{n} t_{2}-\omega_{m} t_{1}\right)}
\end{aligned}
$$

Substituting from equations 16 and 18 gives

$$
\begin{array}{r}
{\left[R_{q q}\left(t_{1}, t_{2}\right)\right]=\sum_{m=0}^{N_{2}-1} \sum_{n=0}^{N_{2}-1}\left\lceil H\left(-\omega_{m}\right)\right\rfloor[\phi]^{T}\{\Gamma\} E\left[\hat{p}_{m}^{*} \hat{p}_{n}\right]} \\
\{\Gamma\}^{T}[\phi]\left\lceil H\left(\omega_{n}\right)\right\rfloor e^{i\left(\omega_{n} t_{2}-\omega_{m} t_{1}\right)}
\end{array}
$$

Note that if the incident acoustic pressure is deterministic, the ensemble average indicated by the expected value in equation 22 is not necessary. If the incident pressure is stochastic, the necessary ensemble averages can be performed ahead of time. For a stationary process, the expected value takes a special form ${ }^{19}$

$$
E\left[\hat{p}_{m}^{*} \hat{p}_{n}\right]=E\left[\hat{p}_{m}^{*} \hat{p}_{n}\right] \delta_{m n}
$$

Then the stationary modal displacement correlation matrix results

$$
\left[R_{q q}(\tau)\right]=\sum_{n=0}^{N_{2}-1} E\left[\left\{\hat{q}_{n}^{*}\right\}\left\{\hat{q}_{n}\right\}^{T}\right] e^{i \omega_{n} \tau}
$$


The stationary modal velocity and acceleration correlation matrices follow

$$
\begin{aligned}
& {\left[R_{\dot{q} \dot{q}}(\tau)\right]=\sum_{n=0}^{N_{2}-1} \omega_{n}^{2} E\left[\left\{\hat{q}_{n}^{*}\right\}\left\{\hat{q}_{n}\right\}^{T}\right] e^{i \omega_{n} \tau}} \\
& {\left[R_{\ddot{q} \ddot{q}}(\tau)\right]=\sum_{n=0}^{N_{2}-1} \omega_{n}^{4} E\left[\left\{\hat{q}_{n}^{*}\right\}\left\{\hat{q}_{n}\right\}^{T}\right] e^{i \omega_{n} \tau}}
\end{aligned}
$$

The correlation matrices with a time lag of zero ( $\tau=0$, so that the diagonal elements are mean square values) can be determined from the modal response power spectrum. An alternative method, which is analytically consistent (so as to avoid normalization factors) involves performing an inverse FFT based upon the above equations. Then the zero time lag correlation matrices correspond to the first element of the inverse transforms. Finally, the stationary response correlation matrices of the physical degrees of freedom result from the modal expansion, equation 10 .

\section{Acoustic Radiation}

The transmitted acoustic pressure radiated by the panel to a point $(x, y, z)$ in the acoustic free-field can be related to the dynamic response of the panel through the use of Rayleigh's integral in the form ${ }^{20}$

$$
p_{t}(x, y, z, t)=\frac{\rho}{2 \pi} \int_{A} \frac{1}{r} \ddot{w}\left(\xi, \eta, t-\frac{r}{c}\right) d A
$$

where $\rho$ is the fluid density, $\ddot{w}$ is the out-of-plane acceleration response of the panel, $\xi$ and $\eta$ are spatial coordinates in the plane of the panel, $r$ is the distance from $(\xi, \eta)$ on the panel to $(x, y, z)$ in the acoustic field, $c$ is the acoustic wave speed, and $t-\frac{r}{c}$ is the retarded time between the two points. Since the physical degrees of freedom are known on an elemental basis

$$
w_{(e)}(\xi, \eta, t) \approx\left\{F_{w}(\xi, \eta)\right\}_{(e)}^{T}\{W(t)\}_{(e)}
$$

the area integral in the expression for the transmitted pressure, equation 26 , is taken to be the sum of element area integrals:

$$
\begin{aligned}
p_{t}(x, y, z, t)=\frac{\rho}{2 \pi} \sum_{k=1}^{n e l} \int_{A_{k}} \frac{1}{r}\left\{F_{w}(\xi, \eta)\right\}_{k}^{T} \\
\left\{\ddot{W}\left(t-\frac{r}{c}\right)\right\}_{k} d A_{k}
\end{aligned}
$$

As before, a discrete spectral analysis can be employed to write

$$
\left\{W\left(t-\frac{r}{c}\right)\right\}=\sum_{n=0}^{N_{2}-1}\left\{\hat{W}_{n}\right\} e^{i \omega_{n}\left(t-\frac{r}{c}\right)}
$$

and

$$
p_{t}(x, y, z, t)=\sum_{n=0}^{N_{2}-1} \hat{p}_{n}(x, y, z) e^{i \omega_{n} t}
$$

As in the panel response section, if the excitation is deterministic, an expression for the radiated pressure spectral amplitude could be developed and used in an inverse FFT to determine the radiated pressure time response. However, for stochastic excitation, a statistical approach must be used.

Substitution of equation 29 into equation 28 results in the following expression for the transmitted pressure

$$
\begin{aligned}
p_{t}(x, y, z, t)=-\frac{\rho}{2 \pi} \sum_{k=1}^{n e l} & \int_{A_{k}} \frac{1}{r}\left\{F_{w}(\xi, \eta)\right\}_{k}^{T} \\
& \sum_{n=0}^{N_{2}-1} \omega_{n}^{2}\left\{\hat{W}_{n}\right\}_{k} e^{i \omega_{n}\left(t-\frac{r}{c}\right)} d A_{k}
\end{aligned}
$$

The transmitted acoustic pressure cross-correlation function follows as

$$
\begin{aligned}
& R_{p p}\left(\vec{x}_{1}, t_{1}, \vec{x}_{2}, t_{2}\right)=\left(\frac{\rho}{2 \pi}\right)^{2} \sum_{k=1}^{n e l} \sum_{l=1}^{n e l} \int_{A_{k}} \int_{A_{l}^{\prime}} \frac{1}{r_{1} r_{2}} \\
& \left\{F_{w}(\xi, \eta)\right\}_{k}^{T} \sum_{m=0}^{N_{2}-1} \sum_{n=0}^{N_{2}-1} \omega_{m}^{2} \omega_{n}^{2} E\left[\left\{\hat{W}_{m}^{*}\right\}_{k}\left\{\hat{W}_{n}\right\}_{l}^{T}\right] \\
& e^{-i \omega_{m}\left(t_{1}-\frac{r_{1}}{c}\right)} e^{i \omega_{n}\left(t_{2}-\frac{r_{2}}{c}\right)}\left\{F_{w}\left(\xi^{\prime}, \eta^{\prime}\right)\right\}_{l} d A_{k} d A_{l}^{\prime}
\end{aligned}
$$

where $r_{1}$ is the distance from $(\xi, \eta)$ on the panel to $\vec{x}_{1}=\left(x_{1}, y_{1}, z_{1}\right)$ in the acoustic field and $r_{2}$ is the distance from $\left(\xi^{\prime}, \eta^{\prime}\right)$ on the panel to $\vec{x}_{2}=\left(x_{2}, y_{2}, z_{2}\right)$ in the acoustic field. Recall that the expected value of the spectral amplitudes takes a simplified form for stationary response:

$$
E\left[\left\{\hat{W}_{m}^{*}\right\}_{k}\left\{\hat{W}_{n}\right\}_{l}^{T}\right]=E\left[\left\{\hat{W}_{m}^{*}\right\}_{k}\left\{\hat{W}_{n}\right\}_{l}^{T}\right] \delta_{m n}
$$

Then the stationary cross-correlation function results $R_{p p}\left(\vec{x}_{1}, t_{1}, \vec{x}_{2}, t_{2}\right) \rightarrow R_{p p}\left(\vec{x}_{1}, \vec{x}_{2}, \tau\right)$, which can be further simplified for zero time lag $(\tau=0$, not to be confused with zero retarded time lag). Thus, the mean square transmitted acoustic pressure at a point $(x, y, z)$ becomes

$$
\begin{array}{r}
\overline{p_{t}^{2}}(x, y, z)=\left(\frac{\rho}{2 \pi}\right)^{2} \sum_{k=1}^{n e l} \sum_{l=1}^{n e l} \int_{A_{k}} \int_{A_{l}^{\prime}} \frac{1}{r_{1} r_{1}^{\prime}}\left\{F_{w}(\xi, \eta)\right\}_{k}^{T} \\
\sum_{n=0}^{N_{2}-1} \omega_{n}^{4} E\left[\left\{\hat{W}_{n}^{*}\right\}_{k}\left\{\hat{W}_{n}\right\}_{l}^{T}\right] e^{i \omega_{n}\left(\frac{r_{1}-r_{1}^{\prime}}{c}\right)} \\
\left\{F_{w}\left(\xi^{\prime}, \eta^{\prime}\right)\right\}_{l} d A_{k} d A_{l}^{\prime}
\end{array}
$$


where $r_{1}$ and $r_{1}^{\prime}$ are distances from the same point $(x, y, z)$ in the acoustic field to different points on the panel, and $\left(r_{1}-r_{1}^{\prime}\right) / c$ is the retarded time difference. The element area integrals can be conveniently evaluated via Gauss-Legendre numerical integration, which precludes the necessity for far-field approximations. However, in the discussions to follow, it will be useful to introduce acoustic far-field assumptions for acoustic intensity calculations. These assumptions entail making the approximations ${ }^{1}$

$$
\frac{1}{r_{1}} \approx \frac{1}{R} \quad \frac{1}{r_{1}^{\prime}} \approx \frac{1}{R}
$$

and

$$
e^{i \omega\left(\frac{r_{1}-r_{1}^{\prime}}{c}\right)} \approx e^{i \omega \frac{\left(\xi^{\prime}-\xi\right) K+\left(\eta^{\prime}-\eta\right) \Lambda}{c}}
$$

where $\mathrm{K}=\sin \theta \cos \phi$ and $\Lambda=\sin \theta \sin \phi$, see figure 2 . Thus, the mean square transmitted acoustic pressure can be written for the far-field as

$$
\begin{gathered}
\overline{p_{t}^{2}}(R, \theta, \phi)=\left(\frac{\rho}{2 \pi R}\right)^{2} \sum_{k=1}^{n e l} \sum_{l=1}^{n e l} \int_{A_{k}} \int_{A_{l}^{\prime}}\left\{F_{w}(\xi, \eta)\right\}_{k}^{T} \\
\sum_{n=0}^{N_{2}-1} \omega_{n}^{4} E\left[\left\{\hat{W}_{n}^{*}\right\}_{k}\left\{\hat{W}_{n}\right\}_{l}^{T}\right] e^{i \omega_{n} \frac{\left(\xi^{\prime}-\xi\right) \mathrm{K}+\left(\eta^{\prime}-\eta\right) \Lambda}{c}} \\
\left\{F_{w}\left(\xi^{\prime}, \eta^{\prime}\right)\right\}_{l} d A_{k} d A_{l}^{\prime}
\end{gathered}
$$

Note that the interpolation functions $F_{w}$ are usually known in terms of element local coordinates. Therefore, the complex exponential function in the integrand of equation 37 must be separated into two components: one which is constant for each element index pair $(k, l)$; the other must be included in the integration over the element areas in local coordinates. The area integrations can be performed numerically, via GaussLegendre numerical integration, but significant reductions in computational time can be realized by performing the integrations in closed form. Other acoustic quantities, such as the acoustic intensity and total radiated power, can be related to this mean square pressure in equation 37 .

The acoustic intensity at a point is equal to the time average of the product of the perturbed pressure and velocity. For a pressure and velocity that are random processes, this temporal average must be taken in the limit as the period $T \rightarrow \infty$ :

$$
\begin{aligned}
I(x, y, z) & =\langle p(x, y, z, t) \vec{u}(x, y, z, t)\rangle \\
& =\lim _{T \rightarrow \infty} \frac{1}{T} \int_{0}^{T} p(x, y, z, t) \vec{u}(x, y, z, t) d t
\end{aligned}
$$

where $\vec{u}(x, y, z, t)$ is the acoustic velocity. Assuming that the processes are ergodic in correlation, this temporal average may be replaced by an expected value

$$
\begin{aligned}
I(x, y, z) & =\langle p(x, y, z, t) \vec{u}(x, y, z, t)\rangle \\
& =E[p(x, y, z, t) \vec{u}(x, y, z, t)]
\end{aligned}
$$

In general, the relationship between the acoustic pressure and velocity is not known. However, in the farfield, the radial velocity is dominant and the propagating waves appear planar. Consequently, the relationship between the acoustic pressure and velocity can be approximated by ${ }^{20,21}$

$$
\vec{u}(R, \theta, \phi, t) \approx u_{r}(R, \theta, \phi, t)=\frac{p(R, \theta, \phi, t)}{\rho c}
$$

where the spatial description has been changed to spherical coordinates for consistency with equation 37. Thus, the far-field transmitted acoustic intensity becomes

$$
I_{t}(R, \theta, \phi)=\frac{1}{\rho c} E\left[p_{t}^{2}(R, \theta, \phi, t)\right]=\frac{\overline{p_{t}^{2}}}{\rho c}
$$

where the mean square pressure has the form given in equation 37 .

The acoustic power incident upon a surface is defined as the integral of the normal acoustic intensity over the surface:

$$
\Pi_{s}=\int_{S} I(R, \theta, \phi) \cdot \hat{n} d S
$$

where $\hat{n}$ is the surface unit normal. Therefore, the total acoustic power transmitted by the plate can be calculated by integrating the acoustic intensity over a far-field hemisphere as follows.

$$
\Pi_{t}=\int_{\phi=0}^{2 \pi} \int_{\theta=0}^{\pi / 2} I_{t}(R, \theta, \phi) R^{2} \sin \theta d \theta d \phi
$$

where the transmitted intensity has the form given in equation 41. This integral must be evaluated numerically, which can be accomplished via Gauss-Legendre numerical integration.

Recall that the incident acoustic pressure consists of obliquely incident plane waves. The incident acoustic intensity is then, from the above discussion,

$$
I_{i}=\frac{\overline{p_{i}^{2}}}{\rho c}
$$


and the incident power is simply the normal acoustic intensity multiplied by the area of the plate:

$$
\Pi_{i}=\frac{\overline{p_{i}^{2}} a b \cos \theta_{i}}{\rho c}
$$

Finally, the transmission loss (TL) from the incident to the receiving acoustic field is defined as

$$
T L=10 \log \left(\frac{\Pi_{i}}{\Pi_{t}}\right)
$$

\section{Results and Discussion}

The results presented in this paper were generated using the following material properties:

$$
\begin{aligned}
& \text { Aluminum } \\
& \mathrm{E}=68.9 \mathrm{GPa}(10 \mathrm{Msi}) \\
& \mathrm{G}=25.8 \mathrm{GPa}(3.75 \mathrm{Msi}) \\
& \nu=0.33 \\
& \rho=2751.4 \mathrm{Kg} / \mathrm{m}^{3}\left(0.2588 \times 10^{-3} \mathrm{lb}-\mathrm{s}^{2} / \mathrm{in}^{4}\right) \\
& \text { Graphite-Epoxy } \\
& \mathrm{E}_{1}=155 \mathrm{GPa}(22.5 \mathrm{Msi}) \\
& \mathrm{E}_{2}=8.07 \mathrm{GPa}(1.17 \mathrm{Msi}) \\
& \mathrm{G}_{12}=4.55 \mathrm{GPa}(0.66 \mathrm{Msi}) \\
& \nu_{12}=0.22 \\
& \rho=1550.07 \mathrm{Kg} / \mathrm{m}^{3}\left(0.1458 \times 10^{-3} \mathrm{lb}_{-} \mathrm{s}^{2} / \text { in. }^{4}\right) \\
& \alpha_{1}=-0.07 \times 10^{-6} /{ }^{\circ} \mathrm{C}\left(-0.04 \times 10^{-6} /{ }^{\circ} \mathrm{F}\right) \\
& \alpha_{2}=30.1 \times 10^{-6} /{ }^{\circ} \mathrm{C}\left(16.7 \times 10^{-6} /{ }^{\circ} \mathrm{F}\right)
\end{aligned}
$$

SMA (Nitinol)

$$
\begin{aligned}
& \mathrm{T}_{\mathrm{s}}=37.78{ }^{\circ} \mathrm{C}\left(100{ }^{\circ} \mathrm{F}\right) \\
& \mathrm{T}_{\mathrm{f}}=62.78(145) \\
& \mathrm{E} \quad \text { From Fig. } 9 \text { of Ref. } 3 \\
& \sigma_{\mathrm{r}}{ }^{*} \quad \text { From Fig. } 8 \text { of Ref. 3 }
\end{aligned}
$$

$$
\begin{aligned}
\mathrm{G} & =24.86 \mathrm{MPa}(3.604 \mathrm{Msi}) \text { for } \mathrm{T}<\mathrm{T}_{\mathrm{s}} \\
& =25.6 \mathrm{MPa}(3.712 \mathrm{Msi}) \text { for } \mathrm{T}>\mathrm{T}_{\mathrm{f}} \\
\nu & =0.3 \\
\rho & =6450 \mathrm{Kg} / \mathrm{m}^{3}\left(0.6067 \times 10^{-3} \mathrm{lb}-\mathrm{s}^{2} / \mathrm{in}^{4}\right) \\
\alpha & =10.26 \times 10^{-6} /{ }^{\circ} \mathrm{C}\left(5.7 \times 10^{-6} /{ }^{\circ} \mathrm{F}\right)
\end{aligned}
$$

A simply supported aluminum panel $(15 \times 6 \times 0.032$ in.) was analyzed for comparison to results presented by Roussos ${ }^{1}$. A comparison of the transmission loss (TL) predicted using each method for an incident plane wave at $\theta_{i}=60^{\circ}$ and $\phi_{i}=0^{\circ}$ is shown in figure 3 . It can be seen that the present method agrees very well with the classical approach for excitation frequencies up to $2 \mathrm{kHz}$. The deviation above $2 \mathrm{kHz}$ is attributable to the modal resolution of the finite element model (element size $1.07 \times 1 \mathrm{in}$.). The radiation predictions are only accurate to within the ability of the finite element model to capture the panel vibration response, as is the case with any other approach.

Transmission loss curves were also generated for simply supported $(0,45,-45,90)_{\mathrm{s}}$ composite panels $(15 \times 6 \times 0.032$ in. $)$ with and without shape memory alloy wire reinforcement. The transmission loss as a function of harmonic excitation frequency is shown in figure 4 for two panels at ambient temperature $\left(70^{\circ} \mathrm{F}\right)$; a graphite-epoxy panel without SMA reinforcement, and a hybrid graphite-epoxy panel with $10 \%$ SMA volume fraction and 5\% initial strain in all layers (SMA inactivated). The shift in the first transmission loss dip at the fundamental frequency, and the moderate TL increase for most of the frequency range above the fundamental frequency, is attributable to the increased mass of the SMA reinforced panel.

The transmission loss for the same hybrid composite panel at $150^{\circ} \mathrm{F}$ and $300^{\circ} \mathrm{F}$ (i.e., SMA activated) is shown in comparison to the panel with inactive SMA in figure 5. It can be seen that at the elevated temperatures the increased stiffness due to tensile inplane recovery forces has greatly increased the fundamental frequency, and thus the fundamental-frequency TL dip. Consequently, there is a significant increase in the transmission loss at low frequencies (below $100 \mathrm{~Hz}$ ). However, at higher frequencies the TL is nearly unchanged. This is due to the fact that the TL is mass controlled at high frequencies. The reduction in the low frequency TL for the hybrid panel at $300^{\circ} \mathrm{F}$, relative to that for the $150^{\circ} \mathrm{F}$ panel, is caused by counteracting compressive inplane forces from thermal expansion effects.

It appears from the above discussion that there are two ways of improving the TL for SMA hybrid composite panels: optimizing the use of the SMA 
fiber reinforcement for maximum stiffening effect with minimal mass addition, and reducing the radiation efficiency of the low order modes (particularly the first). These observations are in agreement with results presented by Liang et al. ${ }^{14}$ and Anders et al. ${ }^{15}$, who have initiated efforts addressing the radiation efficiency problem. The increased flexibility of the finite element structural basis for the present analysis may prove beneficial in future studies aimed at exploring methods of maximizing the SMA recovery effect and altering the vibration mode shapes so that their radiation efficiency is reduced.

\section{Conclusions}

A method has been developed for predicting the vibration response and resulting far-field acoustic radiation for composite panels subject to elevated temperatures and an obliquely incident acoustic excitation consisting of plane waves of arbitrary temporal characteristics. The panel was modeled by a finite element analysis and the radiated field was predicted using Rayleigh's integral. The approach has the ability to include other effects such as shape memory alloy fiber reinforcement, large deflection thermal postbuckling, non-symmetric SMA distribution or lamination, etc.

Analytical results were generated for an aluminum panel subject to harmonic acoustic excitation and compared with a classical analysis for validation of the approach. The two approaches agreed very well. Results were also presented for graphite-epoxy panels with and without shape memory alloy fiber reinforcement. It was found that the main effect of shape memory alloy reinforcement on the radiated acoustic power is a shift in the first transmission loss dip, due to a shift in the panel fundamental frequency. It was observed that further improvements in the panel transmission loss could be realized with more effective use of the SMA reinforcement to maximize the fundamental frequency shift and reduce the radiation efficiency of the fundamental mode.

\section{References}

1. Roussos, L. A.: "Noise Transmission Loss of a Rectangular Plate in an Infinite Baffle," NASA TP-2398, March, 1985.

2. Fuller, C. R.; Rogers, C. A.; and Robertshaw, H. H.: "Control of Sound Radiation with Active/Adaptive Structures," Journal of Sound and Vibration(1992) 157(1), 19-39.
3. Turner, T. L.; Zhong, Z. W.; and Mei, C.: "Finite Element Analysis of the Random Response Suppression of Composite Panels at Elevated Temperatures Using Shape Memory Alloy Fibers," Proceedings of the 35th Structures, Structural Dynamics and Materials Conference, Hilton Head, SC, April 18-21, 1994, pp. 136-146.

4. Cross, W. B.; Kariotis, A. H.; and Stimler, F. J.: "Nitinol Characterization Study," NASA CR1433, 1969.

5. Jackson, C. M.; Wagner, H. J.; and Wasilewski, R. J.: "55-Nitinol The Alloy with a Memory: Its Physical Metallurgy, Properties, and Applications," NASA SP-5110, 1972.

6. Rogers, C. A.; Liang, C.; and Jia, J.: "Behavior of Shape Memory Alloy Reinforced Composite Plates - Part 1: Model Formulation and Control Concepts," Proceedings of the 30th Structures, Structural Dynamics and Materials Conference, Mobile, AL, April 3-5, 1989, pp. 2011-2017.

7. Jia, J.; and Rogers, C. A.: "Formulation of a Mechanical Model of Composites with Embedded SMA Actuators," Proceedings of the 8th Biennial Conference on Failure Prevention and Reliability, Montreal, Canada, September, 17-21, 1989, pp. $203-210$.

8. Baz, A.; and Tampe, L.,: "Active Control of Buckling of Flexible Beams," Proceedings of the 8th Biennial Conference on Failure Prevention and Reliability, Montreal, Canada, September 17-21, 1989, pp. 211-218.

9. Chaudhry, Z.; and Rogers, C. A.: "Bending and Shape Control of Beams Using SMA Actuators," Journal of Intelligent Systems and Structures, Vol. 2, 1991, pp. 581-602.

10. Baz, A.; Iman, K.; and McCoy, J.: "Active Vibration Control of Flexible Beams Using Shape Memory Actuators," Journal of Sound and Vibration, Vol. 140, 1990, pp. 437-456.

11. Srinivasan, A. V.; Cutts, D. G.; and Schetky, L. M.: "Thermal and Mechanical Consideration in Using Shape Memory Alloys to Control Vibrations in Flexible Structures," Metallurgical Transactions A, Vol. 22A, 1991, pp. 623-627.

12. Maclean, B. J.; Patterson, G. J.; and Misra, M. S.: "Modeling of a Shape Memory Integrated Actuator for Vibration Control of Large Space Structures," Journal of Intelligent Materials Systems and Structures, Vol. 2, 1991, pp. 72-94. 
13. Venkatesh, A.; Hilborn, J.; Bidaux, J. E.; and Gotthardt, R.: "Active Vibration Control of Flexible Linkage Mechanisms Using Shape Memory Alloy Fiber-Reinforced Composites," The 1st European Conference on Smart Structures and Materials, Glasgow, UK, May 12-14, 1992, pp. $185-188$.

14. Liang, C.; Rogers, C. A.; and Fuller, C. R.: "Acoustic Transmission and Radiation Analysis of Adaptive Shape Memory Alloy Reinforced Laminated Plates," Journal of Sound and Vibration, Vol. 145, 1991, pp. 23-41.

15. Anders, W. S.; Rogers, C. A.; and Fuller, C. R.: "Vibration and Low Frequency Acoustic Analysis of Piecewise-Activated Adaptive Composite Panels," Journal of Composite Materials, Vol. 26, 1992, pp. 103-120.

16. Locke, J. E.: “A Finite Element Formulation for the Large Deflection Random Response of Thermally Buckled Structures," Ph.D. Dissertation, Old Dominion University, Norfolk, VA, July 1988.

17. Locke, J. E.; and Mei, C.: "A Finite Element Formulation for the Large Deflection Random Response of Thermally Buckled Beams," AIAA Journal, Vol. 28, 1990, pp. 2125-2131.

18. Doyle, J. F.: Static and Dynamic Analysis of Structures, Kluwer Academic Publishers, Dordrecht, The Netherlands, 1991.

19. Nigam, N. C.: Introduction to Random Vibrations, The MIT Press, Cambridge, Massachusetts, 1983 .

20. Pierce, A. D.: Acoustics: An Introduction to its Physical Principles and Applications, Acoustical Society of America, Woodbury, NY, 1989.

21. Temkin, S.: Elements of Acoustics, John Wiley and Sons, New York, NY, 1981.

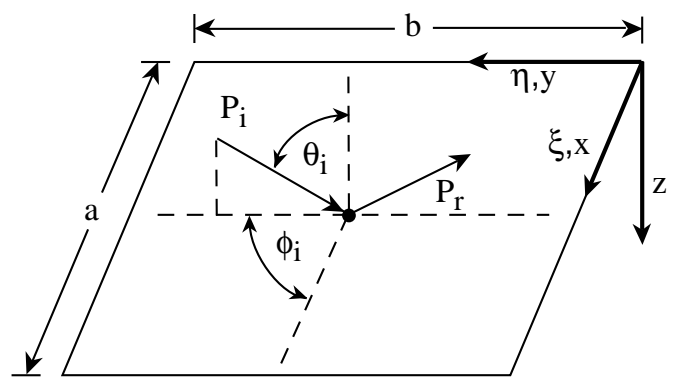

Figure 1: Geometry on incident side of panel (after Roussos $^{1}$ ).

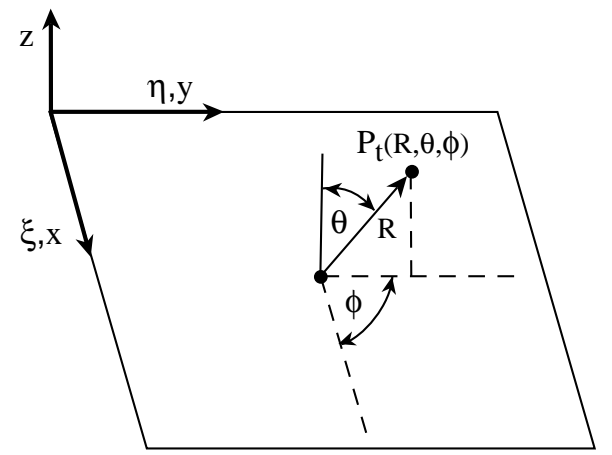

Figure 2: Geometry on radiating side of panel (after Roussos $^{1}$ ).

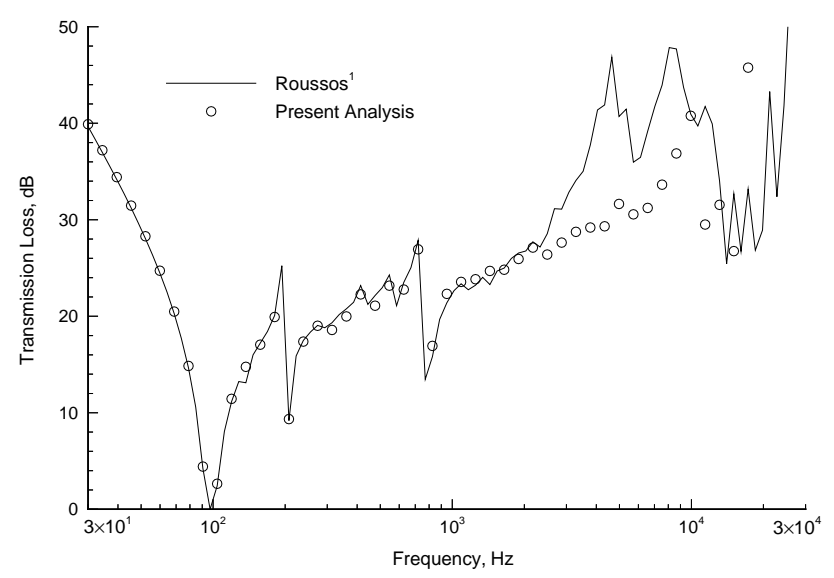

Figure 3: Transmission loss for a $15 \times 6 \times 0.032 \mathrm{in.} \mathrm{alu-}$ minum panel with $\theta_{i}=60^{\circ}$ and $\phi_{i}=0^{\circ}$. 


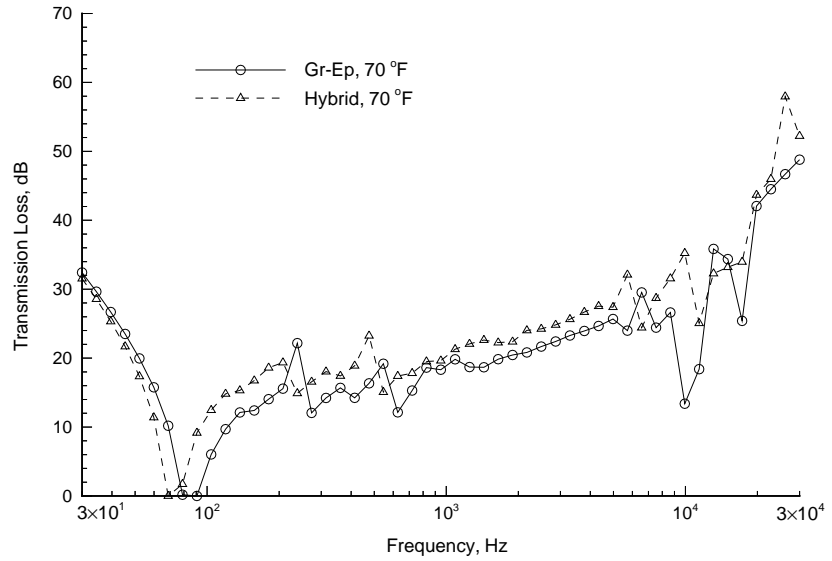

Figure 4: Transmission loss for a $15 \times 6 \times 0.032 \mathrm{in}$. $(0 / 45 /-45 / 90)_{\mathrm{S}}$ composite panel with $\theta_{i}=60^{\circ}$ and $\phi_{i}=0^{\circ}$.

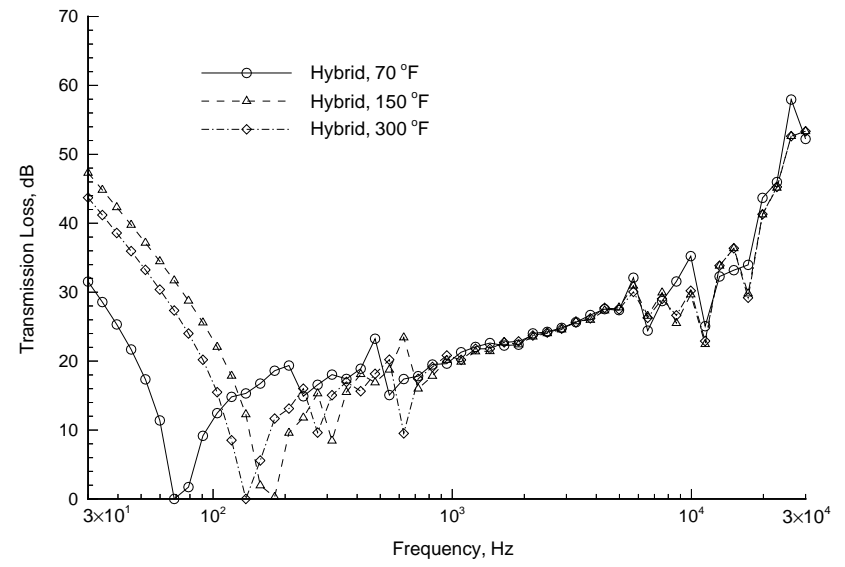

Figure 5: Transmission loss for a $15 \times 6 \times 0.032$ in. $(0 / 45 /-45 / 90)_{\mathrm{s}}$ composite panel with $\theta_{i}=60^{\circ}$ and $\phi_{i}=0^{\circ}$. 